\title{
Processo de Enfermagem para pacientes com Anorexia Nervosa
}

\author{
Nursing Process to patients with Anorexia Nervosa
}

Proceso de Enfermería para pacientes con Anorexia Nerviosa

\author{
Vanessa Pellegrino Toledo', Natália Amorim Ramos', Flávia Wopereis' \\ 'Universidade Estadual de Campinas. Faculdade de Ciências Médicas. Departamento de Enfermagem. Campinas, SP
}

Submissão: $25 / 05 / 2010$

Aprovação: $27 / 07 / 2010$

\section{RESUMO}

Relato de experiência da aplicação do Processo de Enfermagem a uma paciente portadora de Anorexia Nervosa, utilizando histórico, diagnósticos, intervenções e resultados de enfermagem. As intervenções foram fundamentadas nos diagnósticos de enfermagem: distúrbio da imagem corporal, nutrição deseQuilibrada menos Que as necessidades corporais, ansiedade, baixa autoestima crônica, intolerância a atividade, controle ineficaz do regime terapêutico, risco de infecção, volume de lícuidos deficiente e isolamento social. A partir da aplicação do Processo de Enfermagem, os resultados planejados foram alcançados, proporcionando melhor Qualidade de vida, no período em Que permaneceu internada. Os fatores psíuuicos, neurológicos, endócrinos e imunológicos, peculiares na anorexia nervosa, propiciaram a elaboração de um Processo de Enfermagem, Que contribuiu de maneira positiva para a complementação da reabilitação da saúde da mesma.

Descritores: Enfermagem psiQuiátrica; Diagnóstico de enfermagem; Anorexia nervosa; Transtornos alimentares.

ABSTRACT
Experience report on the application of the nursing process (NP) to a female patient with Anorexia Nervosa patient, using patient history, diagnoses, nursing interventions and outcomes. The interventions were based on the nursing diagnoses: body image disturbance, altered nutrition less than body requirements, anxiety, chronic low self-esteem, activity intolerance, ineffective management of therapeutic regimen, risk for infection, fluid volume deficit and social isolation. Based on the application of the Nursing Process, the planned results were achieved, favoring a better Quality of life during the patient's hospitalization time. Mental, neurological, endocrine and immunological factors, which are peculiar in anorexia nervosa cases, permitted the elaboration of an Nursing Process, which positively contributed to complement the patient's health rehabilitation.

Key words: Psychiatric nursing; Nursing diagnosis; Anorexia nervosa; Eating disorders.

\section{RESUMEN}

Relato de experiencia de la aplicación del proceso de enfermería (PE) a una paciente portadora de Anorexia Nerviosa, utilizando histórico, diagnósticos, intervenciones y resultados de enfermería. Las intervenciones fueron basadas en los diagnósticos de enfermería: trastorno de la imagen corporal, nutrición alterada ingesta inferior a las necesidades corporales, ansiedad, autoestima baja crónica, intolerancia a la actividad, manejo ineficaz del régimen terapéutico, riesgo de infección, déficits de volumen de líeuidos y aislamiento social. A partir de la aplicación del Proceso de Enfermería, los resultados planeados fueron alcanzados, proporcionando mejor calidad de vida en el período en Que permaneció hospitalizada. Los factores psíquicos, neurológicos, endócrinos y inmunológicos, peculiares en la anorexia nerviosa, propiciaron la elaboración de un Proceso de Enfermería, Que contribuyó de manera positiva para la complementación de la rehabilitación de su salud.

Descriptores: Enfermería psiQuiátrica; Diagnóstico de enfermería; Anorexia nerviosa; Trastornos de la conducta alimentaria.

AUTOR Vanessa Pellegrino Toledo. Avenida Lauro Correa da Silva, 3805, casa 87, CEP 1348 I-631. Limeira-SP.

CORRESPONDENTEＥ-mail: vtoledo@fcm.unicamp.br 


\section{INTRODUÇÃO}

Estudos clínicos mostram um número crescente de indivíduos Que estão desenvolvendo distúrbios alimentares no ocidente. De acordo com a literatura as causas da anorexia nervosa estão ligadas a fatores psíquicos, neurológicos, endócrinos e imunológicos, Que associam-se à perda da auto estima e sentimentos de desamparo, gerando estresse e impotência sobre o controle da vida ${ }^{(1)}$.

A Anorexia Nervosa é um distúrbio alimentar Que está associado ao aparecimento de incapacidades físicas e psicológicas crônicas, com episódios agudos de complicações médicas ${ }^{(2)}$.

Está presente um inexplicável medo de ganhar peso ou de tornarse obeso, mesmo estando abaixo do peso, além da distorção da imagem corporal. O medo intenso ou mórbido de engordar representa o aspecto psicopatológico central da anorexia(3).

Estudos mostram Que em razão da preocupação excessiva com a imagem corporal, cerca de nove a cada dez pacientes com Anorexia Nervosa são do sexo feminino, já no sexo masculino a incidência é menor pois considera-se um corpo mais musculoso como representação da imagem corporal masculina ideal. A mortalidade dentre os pacientes com Anorexia Nervosa é de $15 \%{ }^{(4)}$.

O prejuízo pessoal e social de indivíduos caracteristicamente jovens, o curso longo e variável, e o prognóstico reservado, reQuerem um planejamento terapêutico eficaz, baseado numa equipe multidisciplinar. Os melhores resultados ocorrem em casos de intervenção precoce durante a adolescência, evitando as formas crônicas e imutáveis das doenças alimentares ${ }^{(2)}$.

Esses pacientes caracterizam-se como indivíduos rígidos, de difícil relacionamento e manipulativos com relação ao tratamento. Tais características provocam inúmeros sentimentos na equipe de enfermagem, Que interferem na Qualidade da assistência prestada ${ }^{(5)}$.

Isto posto, destaca-se Que a identificação dos comprometimentos psíquicos decorrentes da internação psiquiátrica em casos de Anorexia Nervosa é fundamental para Que o enfermeiro possa subsidiar e otimizar o cuidado integral a estes pacientes ${ }^{(6)}$.

A relação terapêutica entre o enfermeiro e o paciente auxilia na implementação do processo de enfermagem psiQuiátrica, para Que efetivamente se possa estabelecer as bases de um planejamento terapêutico Que sustentem a assistência de enfermagem a indivíduos com anorexia nervosa ${ }^{(6)}$.

A abordagem deste estudo baseou-se na assistência de enfermagem. Tendo como pressuposto Que o enfermeiro presta cuidados integrais e contínuos ao paciente psiQuiátrico, desde seu estado mais estável ao mais crítico, julga-se relevante a utilização de um método Que lhe permita dispensar assistência mais Qualificada e organizar suas ações. O Processo de Enfermagem (PE) pode ser definido como aplicação prática de um modelo assistencial de enfermagem na assistência aos pacientes. Este processo é um instrumento metodológico que possibilita identificar, compreender, descrever, explicar e/ou predizer a resposta dos indivíduos aos problemas de saúde ou aos processos vitais, e determinar Que aspectos dessas respostas exigem uma intervenção profissional ${ }^{(7-8)}$.

A enfermagem tem apresentado necessidades de padronização de uma linguagem que pudesse ser entendida e praticada por enfermeiros em vários locais. A partir dessas necessidades começaram a ser criados instrumentos de trabalho Que proporcionam interação dinâmica durante a execução do PE, sendo eles: os sistemas de classificação de Diagnósticos de Enfermagem (North American Nursing Diagnosis Association - NANDA), Classificação das Intervenções de Enfermagem (Nursing Interventions Classification - NIC) e Classificação dos Resultados de Enfermagem (Nursing Outcomes Classification - NOC) ${ }^{(7)}$.

O sistema de classificação de diagnósticos de enfermagem da NANDA é um dos mais conhecidos e divulgados no âmbito mundial. Atualmente trabalha-se com a taxonomia II, com 167 diagnósticos de enfermagem aprovados até o momento ${ }^{(9)}$.

O sistema de classificação de intervenções de enfermagem (NIC) iniciou-se em 1987 e continua em desenvolvimento. Este instrumento emergiu em parte do trabalho realizado pela NANDA. Atualmente, a NIC apresenta 486 intervenções de enfermagem perfazendo um total de atividades superior a doze mil. A NIC nomeia e descreve intervenções executadas na prática clínica em resposta a um diagnóstico de enfermagem ${ }^{(10)}$.

O sistema de classificação de resultados de enfermagem (NOC) vem sendo desenvolvido desde 1991, por um grupo de pesQuisadores também pertencentes ao grupo da NIC. A NOC é uma classificação abrangente e padronizada de resultados da clientela (indivíduo, família ou comunidade) influenciados pela execução de intervenções de enfermagem ${ }^{(11)}$.

A motivação para o presente estudo decorreu da necessidade de compreender mais de perto a complexidade da assistência de enfermagem a pacientes com anorexia nervosa, Que freQuentemente internam em enfermarias psiquiátricas, correndo sério risco de vida e Que apesar de caquéticas, insistem Que estão obesas, provocando repetidos hábitos purgatórios e mantendo regimes alimentares severos para a perda de peso; enfocando o cuidado de enfermagem embasado no conhecimento científico.

Subsidiado por estas Questões, este estudo propõe relatar a experiência de aplicação do processo de enfermagem a uma paciente com anorexia nervosa, internada numa enfermaria de psieuiatria de um hospital escola.

O objetivo deste estudo é relatar a experiência da aplicação do PE a uma paciente com anorexia nervosa utilizando os diagnósticos de enfermagem da Taxonomia II da NANDA, as intervenções de enfermagem da NIC e os resultados de enfermagem da NOC.

\section{METODOLOGIA}

Tratou-se de um relato de experiência, o Qual permitiu realizar investigações em profundidade de um indivíduo, grupo ou instituição ${ }^{(12)}$.

O estudo foi desenvolvido no Hospital de Clínicas (HC) - UNICAMP no município de Campinas, no período de junho a agosto de 2008, junto a paciente com anorexia nervosa internada na unidade de psiquiatria.

Este estudo foi aprovado pelo Comitê de Ética em Pesquisa da instituição em Que foi realizado, respeitando os princípios da Resolução no 196/96, no Que se refere a pesquisas com seres humanos, sob o protocolo $n^{\circ} 007 / 2008$. Foi autorizado pela paciente, mediante a assinatura do Termo de Consentimento Livre e Esclarecido (TCLE), para garantir o anonimato da mesma foi atribuído um pseudônimo designado Violeta.

Aplicou-se o PE nas seguintes etapas: histórico de enfermagem, diagnósticos de enfermagem, planejamento, implementação e 
avaliação da assistência prestada. Para o histórico de enfermagem, utilizou-se o instrumento de coleta de dados Que consta da entrevista psiquiátrica, exame físico, observação e registro das informações, baseada no modelo proposto por Dalgalarrondo (3).

Já de posse dos dados compilados, procedeu-se à análise dos mesmos, à construção dos diagnósticos de enfermagem (de acordo com a Taxonomia II da NANDA), o planejamento, a implementação (de acordo com a NIC) e, por fim, a avaliação, chegando aos resultados (NOC).

\section{RESULTADOS E DISCUSSÃO}

\section{Apresentação do caso}

Violeta, 29 anos, sexo feminino, parda, natural e procedente de Ipiúna/ MG, solteira. Reside no sítio de sua família em uma casa de alvenaria com seus pais e sete irmãos. Relata Que não mantém bom relacionamento com os irmãos e Que um deles cometeu suicídio. Namorou várias vezes, é solteira. Refere não possuir ocupação atual, embora já tenha exercido a profissão de trabalhadora rural. Nega desejo de procurar alguma ocupação, mas realiza tarefas domésticas cotidianas. Relata ter Anorexia Nervosa e reclama que Quando come parece Que a "comida não desce". Foi realizada endoscopia digestiva alta, sem alterações. Provoca vômito constantemente e apesar de monitorada continua praticando este hábito. Diz ter este comportamento há treze anos e que teve início após assistir um filme na televisão no Qual a personagem comia exageradamente e depois vomitava. Refere beber muita água depois de comer, o Que ajudava a provocar vômito. Come de maneira rápida e exagerada, mas depois fica pensando Que vai engordar porQue comeu. Verbaliza que se sente gorda. Refere antecedentes pessoais e familiares de depressão. Nega antecedentes de doenças respiratórias, cardiovasculares e metabólicas. Passava dias em estado de inapetência ingerindo apenas água, tem preferências alimentares por churrasco, maionese e batata frita. Quando Questionada acerca de atividades de lazer declara não possuir desejo de realizá-las. Na enfermaria demonstra interesse para maquiar-se e caminhar pelo pátio. Ao exame psíquico, cuidado pessoal e higiene adequados, usa blusas de frio independente do clima, e gosta de caminhar todo o tempo, para "Queimar calorias". Nível de consciência: desperto, integrando coerentemente aos estímulos ambientais, abertura ocular espontânea, discurso coerente e adeQuado, resposta motora voluntária, obedece a comandos verbais. Orientação temporal, espacial e autopsíquica presente. Atenção voluntária e espontânea. Memória recente, remota, verbal e visual preservadas. Sensopercepção preservada. Pensamento com curso e forma sem alterações, e com conteúdo lógico. Linguagem coerente, falando basicamente o Que lhe é perguntado, com vocabulário mediano. Inteligência com bom rendimento psíquico. Juízo de realidade sem delírios. Não participa das atividades propostas espontaneamente, com exceção a atividades relacionadas à aparência pessoal. Fala apenas euando interrogada, demonstrando pouco interesse na comunicação. Apresenta discurso com traços depressivos, dizendo Que não se importaria em morrer, e Que as pessoas não sentiriam sua falta. Apresenta-se na maioria das vezes, ansiosa. Não se considera doente, pois diz Que a realidade Que vive, com relação aos sintomas e conseQüências de suas atitudes, são "normais" e aceitas socialmente. Ao exame físico, paciente com o peso mensurado de $37.5 \mathrm{~kg}$, altura: $1.49 \mathrm{~m}$, IMC: 16.89 , sendo peso de internação $27.8 \mathrm{~kg}$, devido à anorexia nervosa. Pele de coloração pálida $(+/ 4+)$, anictérica, acianótica, afebril $\left(36.5^{\circ} \mathrm{C}\right)$, ressecada, de turgor diminuído. Mucosas úmidas, descoradas $(2+/ 4+)$. À avaliação dos fâneros apresenta pilificação escassa, alopécia, cabelos de coloração castanha, distribuição uniforme, opacos e Quebradiços. Paciente vigil, lúcida; pupilas de tamanhos normais, isocóricas. Reflexo córneo-palpebral, fotomotor direto e consensual presentes em ambos os olhos. Mantém postura em pé ereta e sentada, alinhamento da cintura pélvica com cintura escapular, locomoção sem auxílio. Apresenta respiração espontânea, eupneica, formato do tórax normal com expansão mantida; à ausculta murmúrios vesiculares presentes, sem ruídos adventícios. Pulso braquial: $65 \mathrm{bpm}$, pressão arterial:112/78 $\mathrm{mmHg}$, bulhas rítmicas normofonéticas sem sopro. Abdome plano, ruídos hidroaéreos normais, ausência de dor à palpação. Gânglios não palpáveis. Paciente relata amenorréia há 10 meses. Globo vesical indolor à palpação e teste de choque lombo-abdominal negativo.

\section{Plano assistencial}

No presente estudo, observou-se Que a paciente necessitava de cuidados tanto físicos, Quanto psíquicos e sociais Que pudessem melhorar o seu Quadro clínico. Deste modo, procurou-se desenvolver um plano de cuidados que estivesse de acordo com a realidade da paciente e da instituição, tornando-a participante e ativa no Que se refere ao planejamento dos seus cuidados.

É importante Que a etapa do planejamento dos cuidados de enfermagem, especialmente em psieuiatria, seja compartilhada com a paciente, garantindo um mínimo de envolvimento acerca da aQuisição de autonomia para Que se possa exercer cada vez mais o controle de sua vida ${ }^{(6)}$.

Embora o foco principal da ação de enfermagem seja a paciente com anorexia, a sua família merece especial atenção. Isto se justifica pela influência Que a família exerce sobre cada um de seus membros, um fator sócio-cultural expresso pela necessidade do indivíduo em seguir o mesmo hábito de vida de seus familiares. Portanto, a mudança no estilo de vida e nos hábitos alimentares da paciente deverá envolver os outros membros da sua família para Que haja um estímulo maior à adesão ao tratamento e ao autocuidado.

O histórico de enfermagem constitui-se no ponto de partida para a elaboração do plano de cuidados. Foram detectados vários problemas reais e potenciais Que, há algum tempo, vinham trazendo prejuízos à vida da paciente, Que se configuraram em nove diagnósticos de enfermagem descritos no Quadro 1 .

Frente a esses diagnósticos, foi elaborado o planejamento da assistência de enfermagem, utilizando as intervenções segundo a classificação da NIC Que pudessem levar a resultados, de acordo com a classificação da NOC, esses resultados demonstraram Que a mesma apresentou melhoras no seu estado físico e mental.

Violeta permaneceu internada na enfermaria por 52 dias. $\mathrm{Na}$ ocasião da internação apresentava IMC $=12$ (baixo peso), na ocasião da alta o IMC $=18,26$. Violeta fez uso também de nutrição parenteral parcial por nove dias.

No que diz respeito ao peso corporal houve uma melhora, porém a paciente permaneceu com baixo peso e comportamentos inadeQuados como: vômitos auto-induzidos, farras alimentares, fuga da enfermaria para comprar alimentos fora do hospital, roubo de alimentos das bolsas 


\begin{tabular}{|c|c|c|}
\hline Diagnóstico de Enfermagem & Resultados Esperados & Prescrições de Enfermagem \\
\hline $\begin{array}{l}\text { Distúrbio da imagem corporal } \\
\text { relacionado à doença, } \\
\text { caracterizado por verbalização } \\
\text { da percepção de Que está gorda. }\end{array}$ & $\begin{array}{l}\text { Deverá ser estimulada a } \\
\text { compreender o distúrbio da } \\
\text { imagem corporal causado } \\
\text { pela doença. }\end{array}$ & $\begin{array}{l}\text { - Esclarecer sobre a doença enfocando a imagem corporal. Incentivar a expressar sentimentos } \\
\text { de como se sente e como vê a si mesma; } \\
\text { - Esclarecer Qualeuer concepção errônea Que ela tenha sobre si mesma, sobre o cuidado, e } \\
\text { sobre as pessoas Que prestam o cuidado; } \\
\text { - Explorar os pontos fortes e os recursos da própria pessoa. }\end{array}$ \\
\hline $\begin{array}{l}\text { Nutrição desequilibrada menos } \\
\text { Que as necessidades corporais } \\
\text { relacionada a fatores } \\
\text { psicológicos, caracterizado por } \\
\text { IMC }=16.89\end{array}$ & $\begin{array}{l}\text { Alcançar o padrão de IMC } \\
\text { aceitável dentro da } \\
\text { normalidade. }\end{array}$ & $\begin{array}{l}\text { - Esclarecer os grupos de alimentos (pirâmide alimentar); } \\
\text { - Explicar o processo de digestão; } \\
\text { - Supervisionar refeições e pós-refeições. }\end{array}$ \\
\hline $\begin{array}{l}\text { Ansiedade relacionada à conflito } \\
\text { inconsciente Quanto a metas de } \\
\text { vida caracterizado por agitação. }\end{array}$ & $\begin{array}{l}\text { Melhora do conforto } \\
\text { psicológico e fisiológico. }\end{array}$ & $\begin{array}{l}\text { - Ficar junto da pessoa; } \\
\text { - Não fazer exigências ou pedir Que ela tome decisões; } \\
\text { - Apoiar os mecanismos de enfrentamento presentes; } \\
\text { - Fornecer feedback sobre a realidade atual, identificar as realizações positivas; } \\
\text { - Falar lenta e calmamente. }\end{array}$ \\
\hline $\begin{array}{l}\text { Baixa auto-estima crônica } \\
\text { relacionado à distúrbio da } \\
\text { imagem corporal, caracterizado } \\
\text { por hesitação em tentar novas } \\
\text { situações. }\end{array}$ & $\begin{array}{l}\text { Deverá identificar os seus } \\
\text { próprios aspectos positivos e } \\
\text { comunicar a libertação dos } \\
\text { sintomas de depressão. }\end{array}$ & $\begin{array}{l}\text { - Prestar apoio, não criticar; } \\
\text { - Esclarecer as distorções, não confrontar; } \\
\text { - Auxiliar a reformular e redefinir as expressões negativas; } \\
\text { - Encorajar bons hábitos físicos (padrões alimentares saudáveis, cuidar do cabelo, maquiar- } \\
\text { se); } \\
\text { - Envolver na terapia de grupo de apoio. }\end{array}$ \\
\hline $\begin{array}{l}\text { Intolerância à atividade } \\
\text { relacionada à fraeueza } \\
\text { generalizada, caracterizada por } \\
\text { relato verbal de frąueza. }\end{array}$ & $\begin{array}{l}\text { Deverá progredir até } \\
\text { conseguir realizar atividades } \\
\text { físicas sem apresentar ou } \\
\text { relatar fadiga ou fraqueza. }\end{array}$ & $\begin{array}{l}\text { - Encorajar exercícios isométricos; } \\
\text { - Aumentar, gradualmente, a tolerância; } \\
\text { - Salientar a importância Que a boa alimentação exerce na força muscular e na prática da } \\
\text { atividade física. }\end{array}$ \\
\hline $\begin{array}{l}\text { Controle ineficaz do regime } \\
\text { terapêutico relacionado à } \\
\text { conflito de decisão, } \\
\text { caracterizado por escolhas da } \\
\text { vida diária ineficazes para atingir } \\
\text { os objetivos da saúde } \\
\text { (compulsão alimentar e indução } \\
\text { ao vômito). }\end{array}$ & $\begin{array}{l}\text { Deverá compreender os } \\
\text { motivos pelos Quais é } \\
\text { necessário adotar } \\
\text { comportamentos saudáveis } \\
\text { para se recuperar. }\end{array}$ & $\begin{array}{l}\text { - Evitar a impressão de estar pressionando; } \\
\text { - Ouvir para descobrir preocupações, não impondo expectativas; } \\
\text { - Explorar o controle anterior bem sucedido de problemas; } \\
\text { - Identificar os fatores Que influenciam no aprendizado; } \\
\text { - Explicar e discutir: processo da doença, regime terapêutico, justificativa do regime, } \\
\text { expectativas e mudanças necessárias no estilo de vida. }\end{array}$ \\
\hline $\begin{array}{l}\text { Risco de infecção relacionado à } \\
\text { desnutrição. }\end{array}$ & $\begin{array}{l}\text { Deverá relatar os fatores } \\
\text { associados a infecção e as } \\
\text { precauções necessárias. }\end{array}$ & $\begin{array}{l}\text { - Encorajar e manter a dieta protéica e calórica na dieta: } \\
\text { - Observar as manifestações clínicas de uma possível infecção na cliente; } \\
\text { - Instruir a cliente e a família tendo em vista as causas e os riscos para infecção. }\end{array}$ \\
\hline $\begin{array}{l}\text { Volume de líquidos deficiente } \\
\text { relacionado com perda ativa de } \\
\text { líeuidos caracterizado por perda } \\
\text { súbita de peso. }\end{array}$ & $\begin{array}{l}\text { Deverá manter a densidade } \\
\text { especifica de urina dentro } \\
\text { dos limites normais. }\end{array}$ & $\begin{array}{l}\text { - Realizar registro de balanço hídrico (lieuido ingerido menos eliminação urinária); } \\
\text { - Pesar diariamente; } \\
\text { - Monitorar a ingestão hídrica, assegurar, no mínimo, 2000ml de líquidos orais a cada } 24 \\
\text { horas; } \\
\text { - Monitorar urina e eletrólitos séricos; } \\
\text { - Fornecer instruções escritas e verbais sobre os líeuidos desejados e as Quantidades, } \\
\text { - Incluir a pessoa/família na manutenção do registro escrito do balanço hídrico. }\end{array}$ \\
\hline $\begin{array}{l}\text { Isolamento social relacionado } \\
\text { com al terações no estado } \\
\text { mental caracterizado por } \\
\text { expressar sentimentos de } \\
\text { rejeição }\end{array}$ & $\begin{array}{l}\text { Deverá ser capaz de } \\
\text { identificar as razões para seu } \\
\text { isolamento social e } \\
\text { desenvolver formas de } \\
\text { aumentar os relacionamentos } \\
\text { significativos }\end{array}$ & $\begin{array}{l}\text { - Promover a interação social } \\
\text { - Encorajar a falar dos seus sentimentos } \\
\text { - Encorajar a formação de um sistema de apoio, mobilizando a família }\end{array}$ \\
\hline
\end{tabular}

\section{Quadro 1. Diagnósticos, resultados esperados e prescrições de enfermagem ${ }^{(9-11)}$.}

de funcionários, além de manipular outros pacientes internados a guardarem as refeições para a mesma. A paciente não acredita estar doente e refere que se acha gorda e barriguda.

Na ocasião da alta, passou 24 horas sem apresentar vômitos induzidos, recebeu orientações da eQuipe de enfermagem e nutrição e fora encaminhada para o grupo de transtornos alimentares no Qual teria reuniões semanais.

Em seus retornos ambulatoriais, Que também são semanais, não obteve melhora de comportamento. Na primeira semana pós-alta, apresentou diversos episódios de farras alimentares com posterior indução do vômito. Após esse período a paciente encontrou-se inapetente, não seguindo, portanto, desde sua alta, a dieta indicada pela nutricionista.

$\mathrm{Na}$ consulta ambulatorial do mês de julho/2008 referiu ter suspendido a medicação para fazer uso de bebida alcoólica. A mãe também refere Que não houve mudança do padrão alimentar anterior à internação.

Quanto à participação no grupo de transtornos alimentares, mantivera-se presente até o mês de agosto/2008.

Pôde-se verificar Que a paciente obteve uma boa evolução do Quadro, no período estudado, aceitando com maior disposição as orientações Quanto aos hábitos purgativos, durante o período em Que permaneceu internada.

Acredita-se que o cuidado é mais adequado quando o enfermeiro 
consegue perceber por meio de sua experiência, de sua habilidade técnica e cognitiva as reais demandas de cuidado e, assim, elabore propostas sistematizadas, individualizadas e prioritariamente articuladas com as necessidades reais do paciente e família ${ }^{(13)}$.

Através do presente estudo, verificou-se Que o PE, Quando aplicado de forma correta e na totalidade de suas fases, representa um grande benefício para o paciente psiQuiátrico além de promover melhorias na organização das atividades de enfermagem, uma assistência mais Qualificada e a conquista da autonomia dos enfermeiros como profissionais de saúde.

\section{CONSIDERAÇÕES FINAIS}

A partir da aplicação do $\mathrm{PE}$, os resultados planejados para a paciente foram alcançados, proporcionando-lhe melhor Qualidade de vida, no período em Que permaneceu internada.

O conhecimento do enfermeiro acerca das alterações apresentadas pela paciente, no Que se refere aos fatores psíquicos, neurológicos, endócrinos e imunológicos, peculiares na anorexia nervosa, propiciaram a elaboração de um PE sistemático, Que contribuiu de maneira positiva para a complementação do processo de reabilitação da saúde da mesma.

Com o surgimento de novas formas de cuidar no contexto da enfermagem psiquiátrica, o enfermeiro psiQuiátrico necessita implementar metodologias de trabalho, pautadas em ações cientificamente comprovadas, deixando de lado o empirismo das condutas rotineiras, com caráter de imposição, controle e vigilância, baseando-se na clínica psiquiátrica através do emprego dos diagnósticos de enfermagem, bem como de outros modelos Que podem ser adotados.

Destaca-se a necessidade de um maior compromisso da família com o plano terapêutico proposto para a paciente após a alta hospitalar, incluindo um projeto de reinserção social, além de um acompanhamento em equipamentos públicos especializados de saúde mental, a fim de prevenir a recidiva do Quadro, e oferecer suporte terapêutico adequado. Vale ressaltar a necessidade do acompanhamento destes casos, já Que no cenário extra-hospitalar, a paciente apresentou muita dificuldade em aderir aos comportamentos positivos para a melhora da saúde, visto Que não há serviços especializados em seu município.

Espera-se Que este estudo contribua para Que os enfermeiros possam refletir Quanto à importância da aplicação do PE como um instrumento metodológico e sistematizado para a melhoria da Qualidade da assistência de enfermagem psieuiátrica, nos diferentes cenários da sua prática profissional. Já Que é um tema Que reQuer um exercício mais aprofundado, na área da enfermagem psiQuiátrica, em decorrência da complexidade dos sinais e sintomas com que se depara, merecendo o destaque de exemplos para nortear as ações de enfermeiros de diferentes instituições tanto públicas Quanto privadas, incluindo o desenvolvimento de novos estudos acerca do PE.

\section{REFERÊNCIAS}

1. Vitale E, Lotito L, Maglie RB. A psychoneuroendocrino-immune approach in the nursing treatment of anorexia and bulimia nervosa. Immunopharmacol Immunotoxicol 2009; 3 I (1): 39-50.

2. Dimitropoulos G, Klopfer K, Lazar L, Schacter R. Caring for a sibling with anorexia nervosa: a Qualitative study. Eur Eat Disord Rev [serial on line] 2009 mar [cited2009jul8]; I0(I 002): [16screens]. Available from: http://www3.interscience. wiley.com/cgi-bin/fulltext/I 22500 I 9 I/PDFSTART

3. Dalgalarrondo P. Psicopatologia e semiologia dos transtornos mentais. São Paulo: Artmed; 2008.

4. Ommen I, Meerwijk EL, Kars M, Elburg A, Meijel B. Effective nursing care of adolescents diagnosed with anorexia nervosa: the patients' perspective. I Clin Nurs [serial on line] 2009 abr [cited 2009 jun 15]; 7(43):[8screens]. Available from: http:// www3.interscience.wiley.com/cgiin/fulltext/I22457284/PDFSTART

5. Grando LH, Rolim MA. Os transtornos da alimentação sob a ótica dos profissionais de enfermagem. Acta Paul Enferm 2006; 19(3): 265-70.

6. Toledo VP. Sistematização da assistência de enfermagem em um serviço de reabilitação psicossocial [tese]. Ribeirão Preto: Escola de Enfermagem de Ribeirão Preto, Universidade de São Paulo; 2004.

7. Chianca TCM, Rocha AM, Pimentel MO. Sistematização da assistência de enfermagem e o sistema único de saúde. In: Anais do $7^{\circ}$. Simpósio Nacional de Diagnósticos de Enfermagem; 2004, 29 abr- I maio; Belo Horizonte (MG), Brasil. Belo Horizonte: ABEn; 2004. p. 24-52.

8. Horta WA. Processo de enfermagem. São Paulo: EPU; 1979.

9. North American Nursing Diagnosis Association. Diagnósticos de enfermagem da NANDA: definição e classificação 2007 2008. Porto Alegre: Artmed; 2008.

10. McCloskey IC, Bulechek GM. Classificação das intervenções de enfermagem (NIC). 3a ed. São Paulo: Artmed; 2004.

11. Johnson M, Maas M, Moorhead S. Classificação dos resultados de enfermagem (NOC). $3^{a}$ ed. São Paulo: Artmed; 2008.

12. Polit DF, Beck CT, Hungler BP. Fundamentos de pesquisa em enfermagem métodos avaliação e utilização. Porto Alegre: Artmed; 2004.

13. Vargas RS, França FCV. Processo de enfermagem aplicado a um portador de cirrose hepática utilizando as terminologias padronizadas NANDA, NIC e NOC. Rev Bras Enferm 2007; 60(3): 348-52. 